\title{
Lower Heart Rate Variability Is Associated with Lower Pulse Pressure Amplification: Role of Obesity
}

\author{
Nicola Di Daniele Manfredi Tesauro Alberto Mascali Valentina Rovella \\ Angelo Scuteri \\ Hypertension Unit, Department of Internal Medicine, Policinico Tor Vergata, Università di \\ Roma Tor Vergata, Rome, Italy
}

\section{Keywords}

Heart rate variability · Pulse pressure amplification · Pulse pressure · Obesity · Artery

\begin{abstract}
Background: Heart rate variability (HRV), pulse pressure amplification, and obesity represent risk factors for cardiovascular events. The aims of the present study are (1) to explore the impact of HRV on pulse pressure amplification and (2) to investigate whether the association between HRV and pulse pressure amplification differs in obese and lean subjects. Methods: A total of 342 patients (age $61 \pm 11$ years) were enrolled. HRV was analyzed concerning both the frequency and time domain as well as concerning the HRV triangular index. Pulse pressure amplification was estimated as the ratio between brachial and carotid pulse pressure, the latter measured with SphygmoCor. Results: Time domain HRV indices were directly correlated with pulse pressure amplification (the lower the HRV indices, the lower the pulse pressure amplification). This association was stronger in obese than in lean subjects after controlling for age and sex. Conclusion: Larger controlled studies are needed to provide a more detailed insight into the relation between HRV and pulse pressure amplification and to determine which pathways are differentially activated in lean and obese subjects.
\end{abstract}

(C) 2017 S. Karger AG, Basel

\section{Introduction}

The pulse wave generated by the heart at each beat travels along the arterial system. Its amplitude (pulse pressure [PP]) has been related to greater cardiovascular (CV) risk, particularly in women [1], and aortic remodeling [2] predisposing to systolic hypertension [3]. At 
a younger age, physiologically, the amplitude (i.e., PP) of the pressure wave increases gradually from the aorta/carotid arteries to the brachial/radial arteries. This phenomenon has been referred to as PP amplification. The lower the central PP and the greater the PP amplification, the more beneficial the effect on the CV system, because of a lower load on the left ventricle. PP amplification decreases with age $[4,5]$. Observational studies also reported that obesity and other classical CV risk factors are associated with reduced PP amplification $[4,5]$.

Heart rate variability (HRV), i.e., the beat-by-beat fluctuations in heart rate (HR) reflecting the autonomic nervous system balance, has emerged as an indicator of CV risk in adults [6, 7]. In general, increased cardiac sympathetic drive is detrimental, while increased cardiac parasympathetic activity is cardioprotective [8]. Conventionally, measures of HRV are in both the time and frequency domains. In the time domain, the square root of the mean squared differences of successive normal-to-normal (NN) intervals (RMSSD), the proportion of beatto-beat (RR) intervals having a difference of $>50 \mathrm{~ms}$ (pNN50), and the HRV index have been shown to be useful indices of vagal activity. In the frequency domain, both low-frequency (LF; $0.04-0.15 \mathrm{~Hz}$ ) and high-frequency (HF; $0.15-0.40 \mathrm{~Hz}$ ) spectral powers have been used as indices of vagal activity. Regardless of the autonomic function selectively explored by those functions (sympathetic versus parasympathetic) [9], lower values of these indices are associated prospectively with death and disability.

Obesity significantly impacts on pulse wave properties [10-14]. In overweight and obese individuals, particularly in children and teenagers, reduced HRV $[15,16]$ has been documented.

The aims of the present study are (1) to explore the impact of HRV on PP amplification and (2) to investigate whether the association between HRV and PP amplification differs in obese and lean subjects.

\section{Methods}

Study Population

A total of 342 outpatients who came to our attention during a campaign for the assessment of CV risk were enrolled in this study. All patients filled in a brief questionnaire concerning their medical history. No blood samples were drawn.

Office brachial blood pressure (BP) was measured by a physician using a mercury sphygmomanometer after the subjects had rested for $10 \mathrm{~min}$, according to standard procedures. Mean BP was defined as diastolic $\mathrm{BP}+1 / 3$ (systolic - diastolic BP).

\section{PP Amplification}

Measurement of central pressures was determined from radial waveforms using the validated SphygmoCor device (AtCor Medical) [17]. All measurements were made in duplicate by a single operator.

PP amplification is usually defined by the ratio between the distal (brachial) PP and the central (aortic) PP. Alternatively, it can be expressed as the difference (in $\mathrm{mm} \mathrm{Hg}$ ) between PP at the distal and PP at the central sites, usually ranging from 5 to $20 \mathrm{~mm} \mathrm{Hg}$. The PP amplification ratio is considered less dependent on calibration errors [18], and, thus, it has been adopted as the primary index for the purposes of the present study.

Assessment of HRV

HRV was measured with the AtCor Medical HRV software to assess sympathetic/parasympathetic autonomic function. A standard 5-min electrocardiogram recording was performed with the patient in supine position, with a regular and calm breathing pattern (5-min resting study) in a quiet room.

Assessment of overall HRV included HRV triangular index (the integral of the density distribution, i.e., number of all NN intervals plotted in a histogram, divided by the maximum of the density distribution). 
Di Daniele et al.: Lower Heart Rate Variability Is Associated with Lower Pulse Pressure Amplification: Role of Obesity

Table 1. Impact of lower and upper levels of indices of HRV on pulse pressure amplification ratio

\begin{tabular}{|c|c|c|c|c|c|c|}
\hline & \multicolumn{3}{|c|}{ Pulse pressure amplification ratio } & \multicolumn{3}{|c|}{$\begin{array}{l}\text { Reduced pulse pressure amplification } \\
\text { ratio, } \%\end{array}$} \\
\hline & 1st quartile & 4th quartile & $p$ & 1st quartile & 4th quartile & $p$ \\
\hline pNN50, \% & $124.4 \pm 1.4$ & $129.1 \pm 1.4$ & 0.02 & 34.5 & 15.5 & 0.01 \\
\hline RMSDD, $\mathrm{m} / \mathrm{s}$ & $125.4 \pm 1.4$ & $131.2 \pm 1.4$ & 0.004 & 32.9 & 14.0 & 0.004 \\
\hline HRV index & $124.6 \pm 1.4$ & $129.9 \pm 1.4$ & 0.008 & 37.8 & 19.1 & 0.004 \\
\hline $\mathrm{LF}$ & $128.8 \pm 1.4$ & $128.5 \pm 1.4$ & 0.83 & 18.4 & 17.5 & 0.88 \\
\hline $\mathrm{HF}$ & $127.0 \pm 1.4$ & $128.8 \pm 1.4$ & 0.36 & 25.7 & 19.0 & 0.33 \\
\hline LF/HF ratio & $128.3 \pm 1.4$ & $126.4 \pm 1.4$ & 0.37 & 19.7 & 23.7 & 0.54 \\
\hline
\end{tabular}

HRV, heart rate variability; pNN50, proportion of RR intervals having a difference of $>50 \mathrm{~ms}$; RMSSD, square root of the mean squared differences of successive NN intervals; LF, power spectral density at the low-frequency range $(0.04-0.15 \mathrm{~Hz})$; $\mathrm{HF}$, power spectral density at the high-frequency range $(0.15-0.4 \mathrm{~Hz})$.

Time domain measures are the means and standard deviations of RR intervals recorded by the continuous electrocardiogram, where NN intervals represent all the RR intervals. The time domain of HRV was evaluated using:

- $\quad$ RMSSD (m/s), which provides an estimate of the short-term components of HRV; the lower this vagal index, the greater the CV burden; and

- $\quad$ pNN50 (\%), which reflects alterations in autonomic function that are primarily vagally mediated and that are virtually independent of circadian rhythms.

The frequency domain of HRV consists of the spectral analysis of a series of consecutive RR intervals in order to quantify sympathetic and vagal influences on the heart. The evaluated parameters were $[9,19]$ :

- $\quad$ power spectral density at the HF range (0.15-0.4 Hz);

- $\quad$ power spectral density at the LF range (0.04-0.15 Hz) - a composite index of cardiac sympathetic and parasympathetic activity; and

- $\quad$ the LF:HF ratio as an indicator of the relative contribution of the sympathetic and parasympathetic components to the autonomic control of the HR.

\section{Statistical Analysis}

All analyses were performed using the SAS package for Windows (version 9.1, Cary, NC, USA). Data are presented as means \pm standard deviations unless otherwise specified. Differences in mean values for each of the measured variables between groups were compared by ANOVA, followed by the Bonferroni test for multiple comparisons. ANCOVA analysis was adopted to compare means across groups after controlling for age and sex. A 2-sided $p$ value $<0.05$ indicated statistical significance.

\section{Results}

The subjects participating in the study were $61 \pm 11$ years old. The prevalence of hypertension and diabetes was 89 and $73 \%$, respectively. Lower values of time domain measures were associated with lower PP amplification (Table 1, left columns) and with a greater proportion of subjects with a reduced PP amplification ratio (defined as the lower quartile of PP amplification ratio distribution within the study population) (Table 1, right columns). No significant difference in PP amplification was observed in subjects with low or high values of indices exploring the HRV frequency domain (Table 1).

Since obesity was more common in subjects with HRV indices in the lower quartile than in those with HRV indices in the upper quartile (on average $4 \%$ greater prevalence in the lower quartiles), we compared lean and obese subjects. Obese subjects were on average 5 
Di Daniele et al.: Lower Heart Rate Variability Is Associated with Lower Pulse Pressure Amplification: Role of Obesity

Table 2. HRV indices in lean and obese subjects

\begin{tabular}{lccc}
\hline & $\begin{array}{l}\text { Control } \\
(n=239)\end{array}$ & $\begin{array}{l}\text { Obese } \\
(n=103)\end{array}$ & $\begin{array}{c}t \text { test } \\
p \text { value }\end{array}$ \\
\hline Age, years & $63.5 \pm 9.3$ & $58.1 \pm 10.8$ & $<0.001$ \\
Men, \% & 60 & 54.4 & $<0.05$ \\
Hypertension, \% & 80.3 & 89.5 & 0.32 \\
Diabetes mellitus, \% & 70.4 & 77.2 & 0.27 \\
Brachial SBP, mm Hg & $140.4 \pm 13.4$ & $142.3 \pm 11.4$ & 0.30 \\
DBP, mm Hg & $77.5 \pm 8.0$ & $79.8 \pm 8.4$ & $<0.05$ \\
MBP, mm Hg & $100.2 \pm 9.0$ & $101.9 \pm 8.3$ & 0.12 \\
Brachial PP, mm Hg & $63.1 \pm 11.9$ & $62.3 \pm 11.4$ & 0.64 \\
HR, bpm & $66.8 \pm 8.7$ & $68.9 \pm 8.8$ & 0.12 \\
Carotid SBP, mm Hg & $130.1 \pm 13.4$ & $129.8 \pm 10.9$ & 0.89 \\
Carotid PP, mm Hg & $52.8 \pm 11.4$ & $49.8 \pm 10.6$ & 0.07 \\
PP amplification ratio & $125 \pm 10$ & $132 \pm 13$ & $<0.0001$ \\
pNN50, \% & $7.3 \pm 8.5$ & $11.7 \pm 14.8$ & $<0.05$ \\
RMSDD, m/s & $36.4 \pm 25.8$ & $43.4 \pm 34.3$ & 0.23 \\
HRV index & $6.7 \pm 2.4$ & $6.8 \pm 2.7$ & 0.76 \\
LF & $1,193 \pm 1,569$ & $992 \pm 1,274$ & 0.69 \\
HF & $619 \pm 799$ & $832 \pm 1,076$ & 0.37 \\
LF/HF ratio & $2.79 \pm 1.74$ & $2.02 \pm 1.37$ & $<0.01$ \\
\hline
\end{tabular}

Values are means \pm standard deviations unless otherwise specified. HRV, heart rate variability; SBP, systolic blood pressure; DBP, diastolic blood pressure; MBP, mean blood pressure; PP, pulse pressure; HR, heart rate; pNN50, proportion of RR intervals having a difference of $>50 \mathrm{~ms}$; RMSSD, square root of the mean squared differences of successive NN intervals; LF, power spectral density at the low-frequency range (0.04-0.15 Hz); HF, power spectral density at the high-frequency range $(0.15-0.4 \mathrm{~Hz})$.

years younger than lean subjects (58.1 \pm 13.2 vs. $63.5 \pm 15.1$ years, respectively) and more likely women (45.6 vs. $41.4 \%$ ).

The PP amplification ratio was greater in obese than in lean subjects (Table 2). Though average HR did not significantly differ between lean and obese subjects, resting HR influenced both time domain HRV and PP amplification. Therefore, to investigate potential differences in the impact of HRV indices on PP amplification between lean and obese subjects, baseline HR was introduced as a covariate together with age and sex. Additional models controlled also for diabetes mellitus and BP levels. As illustrated in Table 3, the impact of HRV indices on PP amplification differed between lean and obese subjects (significant interaction term HRV $\times$ obesity). For example, in lean subjects, being in the lower quartile of RMSSD was associated with an average, significant, $5 \%$ greater PP amplification as compared with lean subjects in the upper quartile of RMSSD. In obese subjects, the opposite effect was observed.

\section{Discussion}

The present study found that (1) time domain HRV indices were directly correlated with the PP amplification ratio (the lower the HRV indices, the lower the PP amplification ratio) and (2) the association was stronger in obese than in lean subjects after controlling for age and sex. The study has 2 major limitations. Subjects were recruited during a local screening campaign for CV disease. Thus, our study population is composed predominantly of high-risk subjects (the prevalence of hypertension and diabetes was 89 and $73 \%$, respectively). Additionally, no blood samples were available, nor detailed information about medication use. 
Di Daniele et al.: Lower Heart Rate Variability Is Associated with Lower Pulse Pressure Amplification: Role of Obesity

Table 3. Least square means ( \pm standard error) of pulse pressure amplification ratio in lean and obese subjects by HRV domains (upper vs. lower quartile)

\begin{tabular}{|c|c|c|c|c|c|c|c|}
\hline & \multicolumn{2}{|c|}{ Lean subjects ( $n=239)$} & \multicolumn{2}{|c|}{ Obese subjects $(n=103)$} & \multicolumn{3}{|c|}{$p$ value } \\
\hline & 1st quartile & 4th quartile & 1st quartile & 4th quartile & HRV & obesity & HRV $\times$ obesity \\
\hline \multicolumn{8}{|c|}{ a Adjusted for age, sex, and heart rate } \\
\hline pNN50, \% & $129.0 \pm 1.7$ & $122.6 \pm 1.5$ & $127.6 \pm 1.8$ & $131.2 \pm 2.0$ & 0.40 & $<0.001$ & $<0.01$ \\
\hline RMSDD, $\mathrm{m} / \mathrm{s}$ & $128.7 \pm 1.4$ & $122.5 \pm 1.5$ & $126.7 \pm 1.8$ & $135.1 \pm 1.9$ & 0.12 & $<0.0001$ & $<0.0001$ \\
\hline HRV index & $128.8 \pm 1.4$ & $122.4 \pm 1.5$ & $128.7 \pm 1.9$ & $129.9 \pm 2.0$ & 0.30 & $<0.001$ & $<0.05$ \\
\hline $\mathrm{LF}$ & $124.0 \pm 1.3$ & $128.2 \pm 1.5$ & $134.1 \pm 2.6$ & $128.9 \pm 1.6$ & 0.10 & $<0.001$ & $<0.05$ \\
\hline $\mathrm{HF}$ & $126.1 \pm 1.6$ & $125.9 \pm 1.4$ & $128.4 \pm 1.7$ & $130.7 \pm 2.0$ & 0.44 & $<0.01$ & 0.48 \\
\hline $\mathrm{LF} / \mathrm{HF}$ ratio & $123.8 \pm 1.4$ & $126.2 \pm 1.6$ & $136.1 \pm 2.9$ & $128.6 \pm 1.7$ & 0.24 & 0.36 & $<0.01$ \\
\hline \multicolumn{8}{|c|}{ b Adjusted for age, sex, heart rate, $M B P$, and diabetes mellitus } \\
\hline pNN50, \% & $128.8 \pm 1.4$ & $122.4 \pm 1.4$ & $127.8 \pm 1.7$ & $130.6 \pm 1.9$ & 0.45 & $<0.001$ & $<0.01$ \\
\hline RMSDD, $\mathrm{m} / \mathrm{s}$ & $128.8 \pm 1.4$ & $122.4 \pm 1.5$ & $127.1 \pm 1.7$ & $133.9 \pm 1.9$ & 0.32 & $<0.0001$ & $<0.001$ \\
\hline HRV index & $129.0 \pm 1.3$ & $122.4 \pm 1.3$ & $128.9 \pm 1.8$ & $130.0 \pm 1.8$ & 0.18 & $<0.001$ & $<0.01$ \\
\hline LF & $124.1 \pm 1.2$ & $127.4 \pm 1.5$ & $133.9 \pm 2.5$ & $129.2 \pm 1.5$ & 0.12 & $<0.0001$ & $<0.05$ \\
\hline $\mathrm{HF}$ & $125.7 \pm 1.5$ & $126.0 \pm 1.3$ & $128.6 \pm 1.6$ & $131.1 \pm 1.9$ & 0.40 & $<0.001$ & 0.44 \\
\hline LF/HF ratio & $124.1 \pm 1.3$ & $125.3 \pm 1.6$ & $135.7 \pm 2.7$ & $129.0 \pm 1.6$ & $<0.05$ & 0.36 & $<0.01$ \\
\hline
\end{tabular}

HRV, heart rate variability; pNN50, proportion of RR intervals having a difference of $>50 \mathrm{~ms}$; RMSSD, square root of the mean squared differences of successive NN intervals; LF, power spectral density at the low-frequency range $(0.04-0.15 \mathrm{~Hz})$; $\mathrm{HF}$, power spectral density at the high-frequency range (0.15-0.4 Hz); MBP, mean blood pressure.

The use of specific antihypertensive drug classes might have a role. In fact, antihypertensive drugs with vasodilating effects increase PP amplification as compared to $\beta$-blockers [19]. Similarly, angiotensin-converting enzyme inhibitors [20] and anti-aldosterone [21] have been reported to positively impact on HRV. Controlling for resting HR may have reduced the limitation deriving from a lack of detailed records about medication use.

An additional limitation may be represented by the adoption of body mass index to define obesity rather than waist circumference, a measure of visceral adiposity and "metabolically active" adipose tissue [13]. In fact, it has been reported that central adiposity, measured by waist circumference, but not overall adiposity, measured by body mass index, was associated with decreased HRV - assessed as standard deviation of successive differences in NN intervals and RMSSD [22].

Nonetheless, the present study calls attention to potential novel pathophysiological pathways that may contribute to arterial aging [23] and may promote CV disease onset and progression $[24,25]$. Larger controlled studies are needed to provide more detailed insight into the role of HRV and PP amplification, an index of arterial aging, and to determine which pathways are differentially activated in lean and obese subjects.

Additional studies will also clarify whether the detrimental effects of obesity on reduced HRV and PP amplification, risk factors for CV disease, may be minimized by active healthy behaviors [26]. For instance, physical activity has been associated with more favorable HRV indices, especially those reflecting increased vagal modulation and reduced sympathetic activity [27, 28]. Similarly, a dose-response relationship between physical activity and improved HRV has been reported, and it is observable even in the presence of heart failure [29]. Thus, behaviors that alter this autonomic imbalance toward a more salubrious profile may serve to prevent or at least minimize the effects of certain risk factors (HRV as well as obesity) for CV events. 
Di Daniele et al.: Lower Heart Rate Variability Is Associated with Lower Pulse Pressure Amplification: Role of Obesity

\section{References}

1 Scuteri A, Cacciafesta M, Di Bernardo MG, De Propris AM, Recchi D, Celli V, Cicconetti P, Marigliano V: Pulsatile versus steady-state component of blood pressure in elderly females: an independent risk factor for cardiovascular disease? J Hypertens 1995;13:185-191.

-2 Scuteri A, Chen CH, Yin FCP, Chih TT, Spurgeon HA, Lakatta EG: Functional correlates of central arterial geometric phenotypes. Hypertension 2001;38:1471-1475.

-3 Farasat SM, Morrell CH, Scuteri A, Ting CT, Yin FC, Spurgeon HA, Chen CH, Lakatta EG, Najjar SS: Pulse pressure is inversely related to aortic root diameter implications for the pathogenesis of systolic hypertension. Hypertension 2008;51:196-120.

4 Segers P, Mahieu D, Kips J, et al; Aslepios Investigators: Amplification of the pressure pulse in the upper limb in healthy, middle-aged men and women. Hypertension 2009;54:414-420.

-5 Wykretowicz A, Rutkowska A, Krauze T, Przymuszala D, Guzik P, Marciniak R, Wysocki H: Pulse pressure amplification in relation to body fatness. Br J Clin Pharmacol 2012;73:546-552.

-6 Schuster AK, Fischer JE, Thayer JF, Mauss D, Jarczok MN: Decreased heart rate variability correlates to increased cardiovascular risk. Int J Cardiol 2016;203:728-730.

7 Thayer JF, Yamamoto SS, Brosschot JF: The relationship of autonomic imbalance, heart rate variability and cardiovascular disease risk factors. Int J Cardiol 2010;14:122-131.

-8 Fisher JP, Young CN, Fadel PJ: Central sympathetic overactivity: maladies and mechanisms. Auton Neurosci 2009;148:5-15.

-9 Heart rate variability: standards of measurement, physiological interpretation and clinical use. Task Force of the European Society of Cardiology and the North American Society of Pacing and Electrophysiology. Circulation 1996;93:1043-1065.

10 Scuteri A, Tesauro M, Rizza S, Iantorno M, Federici M, Lauro D, Campia U, Turriziani M, Fusco A, Cocciolillo G, Lauro R: Endothelial dysfunction and arterial stiffness in normotensive normoglycemic first degree relatives of diabetic patients are independent of the metabolic syndrome. Nutr Metab Cardiovasc Dis 2008;18:349356.

11 Strasser B, Arvandi M, Pasha EP, et al: Abdominal obesity is associated with arterial stiffness in middle-aged adults. Nutr Metab Cardiovasc Dis 2015;25:495-502.

12 Catena C, Colussi G, Frangipane A, et al: Carotid artery stiffness is related to hyperinsulinemia and insulinresistance in middle-aged, non-diabetic hypertensive patients. Nutr Metab Cardiovasc Dis 2015;25:968-974.

13 Scuteri A, Orru' M, Morrell CH, Tarasov K, Schlessinger D, Uda M, Lakatta EG: Associations of large artery structure and function with adiposity: effects of age, gender, and hypertension. The SardiNIA Study. Atherosclerosis 2012;221:189-197.

14 Scuteri A, Cunha PG, Agabiti Rosei E, Badariere J, Bekaert S, Cockcroft JR, Cotter J, Cucca F, De Buyzere ML, De Meyer T, Ferrucci L, Francom O, Gale N, Gillebert TH, Hofman A, Langlois M, Laucevicius A, Laurent S, Mattace Raso FUS, Morrell CH, Muiesan ML, Munnery MM, Navickas R, Oliveira P, Orru' M, Pilia MG, Rietzschel ER, Ryliskyte L, Salvetti M, Schlessinger D, Sousa N, Stefanadis C, Strait J, Van Daele C, Villa I, Vlachopoulos C, Wittemann J, Xaplanteris P, Nilsson PM, Lakatta EG; MARE Consortium: Arterial stiffness and influences of the metabolic syndrome: a cross-countries study. Atherosclerosis 2014;233:654-660.

15 Riva P, Martini G, Rabbia F, et al: Obesity and autonomic function in adolescence. Clin Exp Hypertens 2001;23: 57-67.

16 Nagai N, Matsumoto T, Kita H, Moritani T: Autonomic nervous system activity and the state and development of obesity in Japanese school children. Obes Res 2003;11:25-32.

17 Scuteri A, Morrell CH, Orru’ M, Al Ghatrif M, Saba PS, Terracciano A, Ferreli LA, Loi F, Marongiu M, Pilia MG, Delitala A, Tarasov KV, Schlessinger D, Ganau A, Cucca F, Lakatta EG: Gender specific profiles of white coat and masked hypertension impacts on arterial structure and function in the SardiNIA study. Int J Cardiol 2016;217: 92-98.

18 Avolio A, Van Bortel L, Boutouyrie P, et al: The role of pulse pressure amplification in arterial hypertension: experts' opinion and review of the data. Hypertension 2009;54:375-383.

19 Protogerou AD, Stergiou GS, Vlachopoulos C, Blacher J, Achimastos A: The effect of antihypertensive drugs on central blood pressure beyond peripheral blood pressure. Part II: evidence for specific class-effects of antihypertensive drugs on pressure amplification. Curr Pharm Des 2009;15:272-289.

20 Dias da Silva VJ, Montano N, Salgado HC, Fazan R Jr: Effects of long-term angiotensin converting enzyme inhibition on cardiovascular variability in aging rats. Auton Neurosci 2006;124:49-55.

21 Yee KM, Pringle SD, Struthers AD, et al: Circadian variation in the effects of aldosterone blockade on heart rate variability and QT dispersion in congestive heart failure. J Am Coll Cardiol 2001;37:1800-1807.

-22 Windham BG, Fumagalli S, Ble A, Sollers JJ, Thayer JF, Najjar SS, Griswold ME, Ferrucci L: The relationship between heart rate variability and adiposity differs for central and overall adiposity. J Obes 2012;2012: 149516.

-23 Scuteri A, Najjar SS, Orru' M, Usala G, Piras MG, Ferrucci L, Cao A, Schlessinger D, Uda M, Lakatta EG: The central arterial burden of the metabolic syndrome is similar in men and women: the SardiNIA Study. Eur Heart J 2010; 31:602-613.

24 Huikuri HV, Jokinen V, Syvanne M, Nieminen MS, Airaksinen KE, Ikaheimo MJ, et al: Heart rate variability and progression of coronary atherosclerosis. Arterioscler Thromb Vasc Biol 1999;19:1979-1985. 
25 Lakusic N, Mahovic D, Sonicki Z, Slivnjak V, Baborski F: Outcome of patients with normal and decreased heart rate variability after coronary artery bypass grafting surgery. Int J Cardiol 2013;166:516-518.

-26 Andreoli A, Lauro S, Di Daniele N, et al: Effect of a moderately hypoenergetic Mediterranean diet and exercise program on body cell mass and cardiovascular risk factors in obese women. Eur J Clin Nutr 2008;62:892-897.

27 Swain DP, Franklin BA: Comparison of cardioprotective benefits of vigorous versus moderate intensity aerobic exercise. Am J Cardiol 2006;97:141-147.

-28 Hull SS Jr, Vanoli E, Adamson PB, Verrier RL, Foreman RD, Schwartz PJ: Exercise training confers anticipatory protection from sudden death during acute myocardial ischemia. Circulation 1994;89:548-552.

29 Iellamo F, Manzi V, Caminiti G, Sposato B, Massaro M, Cerrito A, Rosano G, Volterrani M: Dose-response relationship of baroreflex sensitivity and heart rate variability to individually-tailored exercise training in patients with heart failure. Int J Cardiol 2013;166:334-339. 